\title{
STABILITY ANALYSIS OF IMPLICIT DIFFERENCE EQUATIONS UNDER RESTRICTED PERTURBATIONS
}

\author{
VOLKER MEHRMANN* AND DO DUC THUAN ${ }^{\dagger}$
}

\begin{abstract}
The stability analysis for linear implicit $m$-th order difference equations is discussed. We allow the leading coefficient coefficient to be singular, i.e., we include the situation that the system does not generate an explicit recursion. A spectral condition for the characterization of asymptotic stability is presented and computable formulas are derived for the real and complex stability radii in the case that the coefficient matrices are subjected to structured perturbations.

Keywords. Implicit difference equation, asymptotic stability, structured perturbation, stability radius, strangeness-free system, positive system.

Mathematics Subject Classifications: 06B99, 34D99,47A10, 47A99, 65P99.
\end{abstract}

1. Introduction. In this paper we consider linear implicit difference equations, sometimes also termed discrete-time descriptor systems, of the form

$$
A_{m} x^{k+m}+A_{m-1} x^{k+m-1}+\ldots+A_{0} x^{k}=f^{k}, k \in \mathbb{N},
$$

with coefficients $A_{i} \in \mathbb{C}^{n, n}$, and the leading coefficient $A_{m}$ is allowed to be a singular matrix. We prescribe initial conditions

$$
x^{0}=\phi_{0}, x^{1}=\phi_{1}, \ldots, x^{m-1}=\phi_{m-1},
$$

with $\phi_{i} \in \mathbb{C}^{n}$ and we set $\phi=\left[\begin{array}{lll}\phi_{m-1}^{T} & \ldots & \phi_{0}^{T}\end{array}\right]^{T}$.

Difference equations of the form (1.1) arise in the discretization of differentialalgebraic equations, e.g., with backward-difference methods [2, 18, from sampling in dynamical systems [13, 19, or in the context of delay-differential-algebraic systems, 9. 14. For a detailed analysis of first order implicit difference equations and further references, see [3, 4.

The main topic of this paper is to study the stability of the difference equation 1.1), when it is subjected to perturbations. As usual for linear constant coefficient systems, the asymptotic stabilty can be characterized via the eigenvalues of the associated matrix polynomial $P(\lambda)=A_{m} \lambda^{m}+A_{m-1} \lambda^{m-1}+\ldots+A_{1} \lambda+A_{0}$, see [12]. We recall the classical results and extend them to the case of a singular leading coefficient in Section 2 But typically the coefficient functions are not exactly known, since they arise, e.g., from a modeling or system identification process, or as coefficient matrices from a discretization process. Thus, a more realistic scenario for the stability analysis is to analyze the robustness of the asymptotic stability under small perturbations, which may also be structured. This is discussed in Section 3 A problem, however, occurs in the case that the leading coeficient becomes singular under perturbations, because then consistency conditions between initial values and the inhomogeneities arise. If these are not met, then the system may not be solvable. To deal with this problem either a reformulation of the system has to be performed which characterizes the consistency conditions, or the perturbations have to be further restricted, see Section 4

But before we can talk about stability of solutions, we need to introduce a solution concept for 1.1.

\footnotetext{
${ }^{*}$ Institut für Mathematik, MA 4-5, TU Berlin, Strasse des 17. Juni 136, D-10623 Berlin, Germany (mehrmann@math.tu-berlin.de).

${ }^{\dagger}$ School of Applied Mathematics and Informatics, Hanoi University of Science and Technology, 1 Dai Co Viet Str., Hanoi, Vietnam (ducthuank7@gmail.com).
} 
Definition 1.1. A sequence $\left\{x_{\phi}^{k}\right\}_{k \in \mathbb{N}}$ is called a solution of equation 1.1 if $\left\{x_{\phi}^{k}\right\}_{k \in \mathbb{N}}$ satisfies 1.1) for all $k \in \mathbb{N}$. An initial vector $\phi$ is called consistent with 1.1. if the associated initial value problem (1.1) has at least one solution. Equation 1.1. is called regular if for every consistent initial condition $\phi$, the associated initial value problem (1.1) has a unique solution.

With this solvability concept at hand, a solution vector $x_{e} \in \mathbb{C}^{n}$ is called an asymptotic equilibrium of (1.1) if the limit

$$
\lim _{k \rightarrow \infty} f^{k}=\left(A_{m}+A_{m-1}+\ldots+A_{0}\right) x_{e}:=f_{e}
$$

exists. We will employ the following definition of asymptotic stability, see e.g. [8, 21].

Definition 1.2. Consider a regular DAE of the form (1.1). Equation (1.1) is called asymptotically stable if it is regular and the unique solution $\left\{x_{\phi}^{k}\right\}_{k \in \mathbb{N}}$ satisfies

$$
\lim _{k \rightarrow \infty} x_{\phi}^{k}=x_{e}
$$

for all consistent initial conditions $\phi$ such that $\max _{1 \leq i \leq m}\left\|x_{e}-\phi_{i}\right\| \leq \eta$ for some $\eta>0$.

The homogeneous equation

$$
A_{m} x^{k+m}+A_{m-1} x^{k+m-1}+\ldots+A_{0} x^{k}=0, k \in \mathbb{N},
$$

is called asymptotically stable if it is regular and the solution $\left\{x_{\phi}^{k}\right\}_{k \in \mathbb{N}}$ satisfies

$$
\lim _{k \rightarrow \infty} x_{\phi}^{k}=0
$$

for all consistent initial conditions $\phi$ such that $\max _{1 \leq i \leq m}\left\|\phi_{i}\right\| \leq \eta$ for some $\eta>0$,

Having introduced the solvability and asymptotic stability concepts, in the next section we present the characterization of asymptotic stability via spectral conditions.

2. Characterization of asymptotic stability. In this section we recall and extend well known results on the asymptotic stability of implicit difference equations. In the following, we denote the open unit disk in the complex plane by $\mathbb{S}_{1}=\{s \in$ $\mathbb{C}|| s \mid<1\}$.

We first consider the first order case $m=1$.

2.1. First order implicit difference equations. In the first order case of (1.1) the equations take the form

$$
A_{1} x^{k+1}+A_{0} x^{k}=f^{k}, k \in \mathbb{N},
$$

and

$$
A_{1} x^{k+1}+A_{0} x^{k}=0, k \in \mathbb{N},
$$

respectively.

If the leading coefficient $A_{1}$ is invertible, then the well-known theory of linear difference equations [21] can be used to study the system, but even if $A_{1}$ is singular, a complete characterization of solvability is possible and can be carried out via the canonical form of matrix pairs. 
Definition 2.1. A matrix pair $\left(A_{1}, A_{0}\right)$ with $A_{1}, A_{0} \in \mathbb{C}^{n, n}$ is called regular if there exists $\lambda \in \mathbb{C}$ such that $\operatorname{det}\left(\lambda A_{1}-A_{0}\right)$ is different from zero. Otherwise, if $\operatorname{det}\left(\lambda A_{1}-A_{0}\right)=0$ for all $\lambda \in \mathbb{C}$, then we say that $\left(A_{1}, A_{0}\right)$ is singular.

If $\left(A_{1}, A_{0}\right)$ is regular, then a complex number $\lambda$ is called a finite eigenvalue of $\left(A_{1}, A_{0}\right)$ if $\operatorname{det}\left(\lambda A_{1}-A_{0}\right)=0$. The set of all finite eigenvalues of $\left(A_{1}, A_{0}\right)$ is called the finite spectrum of the pair $\left(A_{1}, A_{0}\right)$ and denoted by $\sigma\left(A_{1}, A_{0}\right)$. If $A_{1}$ is singular and the pair is regular, then we say that $\left(A_{1}, A_{0}\right)$ has the eigenvalue $\infty$.

Regular pairs $\left(A_{1}, A_{0}\right)$ can be transformed to Weierstra $\beta$ canonical form, see [7. 11, 18, i.e., there exist nonsingular matrices $W, T \in \mathbb{C}^{n, n}$ such that

$$
A_{1}=W\left[\begin{array}{cc}
I_{r} & 0 \\
0 & N
\end{array}\right] T^{-1}, A_{0}=W\left[\begin{array}{cc}
J & 0 \\
0 & I_{n-r}
\end{array}\right] T^{-1}
$$

where $I_{r}, I_{n-r}$ are identity matrices, $J \in \mathbb{C}^{r, r}$ and $N \in \mathbb{C}^{(n-r),(n-r)}$ are matrices in Jordan canonical form and $N$ is nilpotent. If $A_{1}$ is invertible, then $r=n$, i.e., the second diagonal block does not occur.

Definition 2.2. Consider a regular pair $\left(A_{1}, A_{0}\right)$ with $A_{1}, A_{0} \in \mathbb{C}^{n, n}$ in Weierstraß form (2.3). If $r<n$ and $N$ has nilpotency index $\nu \in \mathbb{N}$, i.e., $N^{\nu}=0, N^{i} \neq 0$ for $i=1,2, \ldots, \nu-1$, then $\nu$ is called the index of the pair $\left(A_{0}, A_{1}\right)$ and we write $\operatorname{ind}\left(A_{1}, A_{0}\right)=\nu$. If $r=n$ then the pair has index $\nu=0$.

The general theory of existence and uniqueness (even for variable coefficients) has been been carried out in [3, 4, here we proceed with the regular case.

If the pair $\left(A_{1}, A_{0}\right)$ is regular, and if $\hat{\lambda} \in \mathbb{C}$ is such that $\operatorname{det}\left(\hat{\lambda} A_{1}+A_{0}\right) \neq 0$, then setting

$$
\hat{A}_{1}=\left(\hat{\lambda} A_{1}+A_{0}\right)^{-1} A_{1}, \hat{A}_{0}=\left(\hat{\lambda} A_{1}+A_{0}\right)^{-1} A_{0}, \hat{f}^{k}=\left(\hat{\lambda} A_{1}+A_{0}\right)^{-1} f^{k},
$$

it is easy to see that $\hat{A}_{1} \hat{A}_{0}=\hat{A}_{0} \hat{A}_{1}$ and equation 2.1 has the same solution set as

$$
\hat{A}_{1} x^{k+1}+\hat{A}_{0} x^{k}=\hat{f}^{k}
$$

for which an explicit solution formula exists, which uses projectors based on Drazin inverses, see [6]. Let for a matrix $M \in \mathbb{C}^{n, n}$ the Jordan form be given by

$$
M=T^{-1} J T, \quad J=\left[\begin{array}{cc}
C & 0 \\
0 & N
\end{array}\right],
$$

where $C$ is nonsingular and $N$ is nilpotent, then the Drazin inverse of $M$ is defined as

$$
M^{D}=T^{-1}\left[\begin{array}{cc}
C^{-1} & 0 \\
0 & 0
\end{array}\right] T \text {. }
$$

Using the Drazin inverses $\hat{A}_{1}^{D}, \hat{A}_{0}^{D}$ it has been shown in [4, 6] that if 2.4 (with initial condition $\left.x^{0}=\phi_{0}\right)$ is uniquely solvable, then it has the explicit solution

$$
\begin{gathered}
x^{k}=\left(-\hat{A}_{1}^{D} \hat{A}_{0}\right)^{k} \hat{A}_{1}^{D} \hat{A}_{1} \phi_{0}+\sum_{j=0}^{k-1}\left(-\hat{A}_{1}^{D} \hat{A}_{0}\right)^{k-1-j} \hat{A}_{1}^{D} \hat{f}^{j} \\
-\left(I-\hat{A}_{1}^{D} \hat{A}_{1}\right) \sum_{i=0}^{\nu-1}\left(-\hat{A}_{0}^{D} \hat{A}_{1}\right)^{i} \hat{A}_{0}^{D} \hat{f}^{k+i} .
\end{gathered}
$$


By taking $k=0$, the following formula presents a condition for the consistency of the initial condition with respect to the right hand side sequence

$$
\left(I-\hat{A}_{1}^{D} \hat{A}_{1}\right)\left(\phi_{0}+\sum_{i=0}^{\nu-1}\left(-\hat{A}_{0}^{D} \hat{A}_{1}\right)^{i} \hat{A}_{0}^{D} \hat{f}^{i}\right)=0 .
$$

We also observe that if $\nu>1$ then the system is non-causal, i.e., the solution $x^{k}$ depends on $f^{k}, \ldots, f^{k+\nu-1}$.

Using the explicit solution, we immediately have a characterization of asymptotic stability.

THEOREM 2.3. Consider the difference equations (2.1) and (2.2). If the pair $\left(A_{1}, A_{0}\right)$ is regular and the initial value $\phi_{0}$ is consistent, then the following statements are equivalent.

1) Equation 2.1 is asymptotically stable;

2) Equation 2.2 is asymptotically stable;

3) $\sigma\left(A_{1},-A_{0}\right) \subset \mathbb{S}_{1}$.

Proof. It is obvious that 1) implies 2). To show that 2) implies 3), we employ the solution formula (2.5) and for (2.2) we obtain the solution

$$
x^{k}=\left(-\hat{A}_{1}^{D} \hat{A}_{0}\right)^{k} \hat{A}_{1}^{D} \hat{A}_{1} \phi_{0}
$$

Since we can vary the consistent initial condition $\phi_{0}$ in the set of consistent initial conditions, it follows from 2.6 that $\hat{A}_{1}^{D} \hat{A}_{1} \phi_{0}=\phi_{0}$ and thus asymptotic stability implies that $\lim _{k \rightarrow \infty}\left(-\hat{A}_{1}^{D} \widehat{\left.A_{0}\right)^{k}}=0\right.$, which holds if and only if $\sigma\left(-\hat{A}_{1}^{D} \hat{A}_{0}\right) \subset \mathbb{S}_{1}$. Considering the Weierstraß canonical form 2.3), one obtains that

$$
-\hat{A}_{1}^{D} \hat{A}_{0}=T\left[\begin{array}{cc}
-J & 0 \\
0 & 0
\end{array}\right] T^{-1}
$$

Thus, $\sigma\left(-\hat{A}_{1}^{D} \hat{A}_{0}\right)=\sigma(I,-J)=\sigma\left(A_{1},-A_{0}\right)$ and the claim follows.

To prove that 3$)$ implies 1$)$, we partition

$$
T^{-1} x^{k}=\left[\begin{array}{c}
y^{k} \\
z^{k}
\end{array}\right], W^{-1} f^{k}=\left[\begin{array}{c}
f_{1}^{k} \\
f_{2}^{k}
\end{array}\right], W^{-1} f_{e}=\left[\begin{array}{c}
f_{1 e} \\
f_{2 e}
\end{array}\right],
$$

where $y^{k}, f_{1}^{k}, f_{1 e} \in \mathbb{C}^{r}$. Then, equation 2.1) is equivalent to

$$
\begin{aligned}
y^{k+1}+J y^{k} & =f_{1}^{k}, \\
N z^{k+1}+z^{k} & =f_{2}^{k},
\end{aligned}
$$

with $\lim _{k \rightarrow \infty} f_{1}^{k}=f_{1 e}$ and $\lim _{k \rightarrow \infty} f_{2}^{k}=f_{2 e}$. Since $\sigma(-J)=\sigma\left(A_{0},-A_{1}\right) \subset \mathbb{S}_{1}$, by the theory of difference equations [21, 24], the solution sequence $\left\{y^{k}\right\}_{k \in \mathbb{N}}$ satisfies

$$
\lim _{k \rightarrow \infty} y^{k}=y_{e}=\left(I_{r}+J\right)^{-1} f_{1 e}
$$

Since $N$ is nilpotent, we have $N^{D}=0$ and hence, by 2.5 ,

$$
z^{k}=\sum_{i=0}^{\nu-1}(-1)^{i} N^{i} f^{k+i},
$$


which implies that

$$
\lim _{k \rightarrow \infty} z^{k}=\sum_{i=0}^{\nu-1}(-1)^{i} N^{i} f_{2 e}=\left(N+I_{n-r}\right)^{-1} f_{2 e} .
$$

Thus,

$$
\begin{aligned}
\lim _{t \rightarrow \infty} x^{k} & =T \lim _{k \rightarrow \infty}\left[\begin{array}{l}
y^{k} \\
z^{k}
\end{array}\right]=T\left[\begin{array}{cc}
\left(I_{r}+J\right)^{-1} & 0 \\
0 & \left(N+I_{n-r}\right)^{-1}
\end{array}\right]\left[\begin{array}{l}
f_{1 e} \\
f_{2 e}
\end{array}\right] \\
& =\left(A_{1}+A_{0}\right)^{-1} f_{e}=x_{e}
\end{aligned}
$$

and asymptotic stability of 2.1 follows.

As a consequence of the presented results, for regular systems (1.1) the asymptotic stability is characterized by the finite eigenvalues of $\left(A_{1}, A_{0}\right)$ being inside the unit disk, while the index of the equation $\nu$ is not important. However, if the index of the pair $\left(A_{1}, A_{0}\right)$ is larger than 1 , then there are consistency relations between the right hand side and the initial conditions, which may prevent solvability.

2.2. Higher order implicit difference equations. Using the classical concepts of turning high order difference equations into first order difference equations [12], we can immediately extend the results of Subsection 2.1 to higher order difference equations. Introducing the matrix polynomial

$$
P(\lambda)=A_{m} \lambda^{m}+A_{m-1} \lambda^{m-1}+\ldots+A_{1} \lambda+A_{0}
$$

and denoting the finite roots of $P$ by $\sigma(P)=\{\lambda \mid \operatorname{det}(P(\lambda))=0\}$, we have the following result.

THEOREM 2.4. Consider the difference equations (1.1) and (1.5) and assume that (1.1) is regular and that the intial condition is consistent. Then, the following statements are equivalent

1) Equation 1.1 is asymptotically stable;

2) Equation 1.5 is asymptotically stable;

3) $\sigma(P) \subset \mathbb{S}_{1}$.

Proof. Introduce the companion representation [12] of the difference equation, i.e., the block matrices

$$
\mathcal{A}_{1}:=\left[\begin{array}{cccc}
A_{m} & 0 & \ldots & 0 \\
0 & -I_{n} & \ldots & 0 \\
\vdots & \vdots & \ddots & \vdots \\
0 & 0 & \ldots & -I_{n}
\end{array}\right], \mathcal{A}_{0}:=\left[\begin{array}{cccc}
A_{m-1} & \ldots & A_{1} & A_{0} \\
I_{n} & \ldots & 0 & 0 \\
\vdots & \ddots & \vdots & \vdots \\
0 & \ldots & I_{n} & 0
\end{array}\right] \in \mathbb{C}^{n m, n m}
$$

and the block vectors

$$
X^{k}:=\left[\begin{array}{c}
x^{k+m-1} \\
x^{k+m-2} \\
\vdots \\
x^{k}
\end{array}\right], X_{e}:=\left[\begin{array}{c}
x_{e} \\
x_{e} \\
\vdots \\
x_{e}
\end{array}\right], F^{k}:=\left[\begin{array}{c}
f^{k} \\
0 \\
\vdots \\
0
\end{array}\right] \in \mathbb{C}^{n m} .
$$

Then equation 1.1 is equivalent to

$$
\mathcal{A}_{1} X^{k+1}+\mathcal{A}_{0} X^{k}=F^{k}
$$


and equation 1.5 is equivalent to

$$
\mathcal{A}_{1} X^{k+1}+\mathcal{A}_{0} X^{k}=0 .
$$

It is then obvious that $\lim _{k \rightarrow \infty} X^{k}=X_{e}$ if and only if $\lim _{k \rightarrow \infty} x^{k}=x_{e}$. Thus 1.1 is asymptotically stable if and only if 2.8 is asymptotically stable, and an analogous relation holds for (1.5) and 2.9). Moreover, from the theory of matrix polynomials [12, we immediately have that the pair $\left(\mathcal{A}_{1}, \mathcal{A}_{0}\right)$ is singular if and only if $P(\lambda)$ is singular, and that $\sigma\left(\mathcal{A}_{1}, \mathcal{A}_{0}\right)=\sigma(P)$ and thus the assertion follows from Theorem 2.3

口

Since the asymptotic stability of the inhomogeneous system (1.1) and the homogeneous system 1.5 are equivalent, in the following we only consider (1.5).

It has been shown in [23], that for any matrix tuple $\left(A_{m}, A_{m-1}, \ldots, A_{0}\right)$, there always exists a nonsingular matrix $W \in \mathbb{C}^{n, n}$ such that

$$
W^{-1} A_{m}=\left[\begin{array}{c}
A_{m}^{(0)} \\
0 \\
0 \\
\vdots \\
0
\end{array}\right], W^{-1} A_{m-1}=\left[\begin{array}{c}
A_{m-1}^{(0)} \\
A_{m-1}^{(1)} \\
0 \\
\vdots \\
0
\end{array}\right], \ldots, W^{-1} A_{0}=\left[\begin{array}{c}
A_{0}^{(0)} \\
A_{0}^{(1)} \\
A_{0}^{(2)} \\
\vdots \\
A_{0}^{(m)}
\end{array}\right],
$$

where $A_{m}^{(0)}, A_{m-1}^{(0)}, \ldots, A_{0}^{(0)} \in \mathbb{C}^{d_{0}, n}, A_{m-1}^{(1)}, \ldots, A_{0}^{(1)} \in \mathbb{C}^{d_{1}, n}, \ldots, A_{0}^{(m)} \in \mathbb{C}^{d_{m}, n}$ with $d_{0}+d_{1}+\ldots+d_{m}=n$ and the blocks $A_{m}^{(0)}, A_{m-1}^{(1)}, \ldots, A_{1}^{(m-1)}$ have full row rank. Furthermore, if 1.5 is regular, then also $A_{m}^{(0)}$ has full row rank and the matrix

$$
\widehat{A}_{m}:=\left[\begin{array}{c}
A_{m}^{(0)} \\
\vdots \\
A_{0}^{(m)}
\end{array}\right]
$$

has full rank. Thus equation 1.5 can be scaled by $W^{-1}$ to obtain

$$
\begin{aligned}
A_{m}^{(0)} x^{k+m}+A_{m-1}^{(0)} x^{k+m-1}+\ldots+A_{0}^{(0)} x^{k} & =0, \\
A_{m-1}^{(1)} x^{k+m-1}+\ldots+A_{0}^{(1)} x^{k} & =0, \\
& \vdots \\
A_{0}^{(m)} x^{k} & =0 .
\end{aligned}
$$

Shifting the index in the $i$-th equation by $i-1$, we obtain

$$
\begin{aligned}
A_{m}^{(0)} x^{k+m}+A_{m-1}^{(0)} x^{k+m-1}+\ldots+A_{0}^{(0)} x^{k} & =0 \\
A_{m-1}^{(1)} x^{k+m}+\ldots+A_{0}^{(1)} x^{k+1} & =0 \\
& \vdots \\
A_{0}^{(m)} x^{k+m} & =0,
\end{aligned}
$$

Following the concept of strangeness-index in [14, 18, 23, we make the following definition. 
Definition 2.5. Equation (1.5) is called strangeness-free if there exists a nonsingular matrix $W \in \mathbb{C}^{n, n}$ that transforms the matrix tuple $\left(A_{m}, A_{m-1}, \ldots A_{0}\right)$ to the form (2.12) such that the matrix $\widehat{A}_{m}$ in 2.11) is invertible.

It is easy to show that, although the transformed form (2.12) is not unique (any nonsingular matrix that operates blocks-wise in the block-rows can be applied), the strangeness-free property is invariant under the choice of $W$. If 1.5 is strangenessfree, then introducing

$$
\widehat{A}_{m-1}:=\left[\begin{array}{c}
A_{m-1}^{(0)} \\
\vdots \\
A_{0}^{(m-1)} \\
0
\end{array}\right], \ldots, \widehat{A}_{0}:=\left[\begin{array}{c}
A_{0}^{(0)} \\
0 \\
\vdots \\
0
\end{array}\right]
$$

the implicit system (2.12) is equivalent to the linear difference equation

$$
x^{k+m}=-\widehat{A}_{m}^{-1} \widehat{A}_{m-1} x^{k+m-1}-\ldots-\widehat{A}_{m}^{-1} \widehat{A}_{0} x^{k},
$$

which admits a unique solution that satisfies the consistent initial condition 1.2 .

REMARK 2.6. Suppose that equation (1.5) is strangeness-free and $W$ and $W$ are two nonsingular matrices that both transform the coefficients of the equation to the form 2.10). Let $\widehat{A}_{i}^{(j)}$ be the transformed blocks corresponding to $\widehat{W}$. Introduce the block matrix $R=W^{-1} \widehat{W}$ and let $R=\left(R_{j}^{(i)}\right)$ with $R_{j}^{(i)} \in \mathbb{C}^{d_{i}, d_{j}}$. Then, we have

$$
R\left[\begin{array}{c}
\widehat{A}_{m}^{(0)} \\
0 \\
0 \\
\vdots \\
0
\end{array}\right]=\left[\begin{array}{c}
A_{m}^{(0)} \\
0 \\
0 \\
\vdots \\
0
\end{array}\right], R\left[\begin{array}{c}
\widehat{A}_{m-1}^{(0)} \\
\widehat{A}_{m-1}^{(1)} \\
0 \\
\vdots \\
0
\end{array}\right]=\left[\begin{array}{c}
A_{m-1}^{(0)} \\
A_{m-1}^{(1)} \\
0 \\
\vdots \\
0
\end{array}\right], \ldots, R\left[\begin{array}{c}
\widehat{A}_{0}^{(0)} \\
\widehat{A}_{0}^{(1)} \\
\widehat{A}_{0}^{(2)} \\
\vdots \\
\widehat{A}_{0}^{(m)}
\end{array}\right]=\left[\begin{array}{c}
A_{0}^{(0)} \\
A_{0}^{(1)} \\
A_{0}^{(2)} \\
\vdots \\
A_{0}^{(m)}
\end{array}\right],
$$

and it is easy to verify that $R$ is a block upper-triangular matrix, i.e., $R_{j}^{(i)} \mid 0 \leq j<i \leq$ $m$ are zero blocks. Since $R$ is nonsingular, the diagonal blocks $R_{i}^{(i)}, i=0,1, \ldots, m$, are nonsingular. Thus, $\widehat{W}=W R$ with

$$
R=\left[\begin{array}{cccc}
R_{0}^{(0)} & R_{1}^{(0)} & \ldots & R_{m}^{(0)} \\
0 & R_{1}^{(1)} & \ldots & R_{m}^{(1)} \\
\vdots & \ddots & \ddots & \vdots \\
0 & \ldots & 0 & R_{m}^{(m)}
\end{array}\right] .
$$

As in the first order case, asymptotic stability is characterized by the finite eigenvalue of $P(\lambda)$ being in the open unit disk, while the part associated with the infinite eigenvalues may create extra consistency and solvability conditions.

2.3. Positive systems. In order to compute stability radii under real perturbations, we will need the concept of positive systems. In this subsection we introduce some further notation and characterize positivity of a system, see e.g. [1]. For matrices $B=\left[b_{i j}\right], C=\left[c_{i j}\right] \in \mathbb{R}^{l, q}$ the inequality $B \geq C$ is to be interpreted as $b_{i j} \geq c_{i j}$ for all $1 \leq i \leq l, 1 \leq j \leq q$, the set of all nonnegative matrices in $\mathbb{R}^{l, q}$ is denoted by $\mathbb{R}_{+}^{l, q}$, 
and the set of all nonpositive matrices by $\mathbb{R}_{-}^{l, q}$. Denoting the componentwise absolute value for a matrix $P \in \mathbb{R}^{l, q}$ by $|P|=\left(\left|p_{i j}\right|\right)$, for arbitrary matrices $B, C \in \mathbb{C}^{l, q}$ we have the inequalities

$$
|B+C| \leq|B|+|C|,|B C| \leq|B||C| .
$$

For any $B \in \mathbb{C}^{l, l}$, the spectral radius of $B$ is denoted by $\rho(B)=\max \{|\lambda| \mid \lambda \in \sigma(B)\}$, where $\sigma(B)=\left\{s \in \mathbb{C} \mid \operatorname{det}\left(s I_{l}-B\right)=0\right\}$. The spectral radius has the monotonicity property that for all $C \in \mathbb{C}^{l, l}, B \in \mathbb{R}_{+}^{l, l}$ if $|C| \leq B$ then $\rho(C) \leq \rho(|C|) \leq \rho(B)$. A norm $\|\cdot\|$ on $\mathbb{C}^{l}$ is said to be monotonic, if $|x| \leq|y|$ implies that $\|x\| \leq\|y\|$ for all $x, y \in \mathbb{C}^{l}$. It is easy to see that every $p$-norm on $\mathbb{C}^{l}$ is monotonic. An operator norm $\|\cdot\|$ that is induced by a monotonic vector norm then has the monotonicity property that for all $C \in \mathbb{C}^{l, l}, B \in \mathbb{R}_{+}^{l, l}$ with $|C| \leq B$ we have $\|C\| \leq\||C|\| \leq\|B\|$. Using this notation, we give a definition of positivity for system (1.5).

Definition 2.7. System 1.5 is called positive if for any consistent initial condition $\phi \in \mathbb{R}_{+}^{n m}$ the corresponding solution $\left\{x_{\phi}^{k}\right\}_{k \in \mathbb{N}}$ satisfies $x_{\phi}^{k} \in \mathbb{R}_{+}^{n}$ for all $k \in \mathbb{N}$.

We have an immediate extension of the results in $[22$.

Proposition 2.8. If 1.5 is strangeness-free, then it is positive if and only if for the matrices defined in 2.2 we have $\widehat{A}_{m}, \widehat{A}_{m-1}, \ldots, \widehat{A}_{0} \in \mathbb{R}_{-}^{n, n}$. Moreover, if 1.5 is positive and asymptotically stable then

$$
\rho\left(-\widehat{A}_{m}^{-1} \widehat{A}_{m-1}-\ldots-\widehat{A}_{m}^{-1} \widehat{A}_{0}\right)<1 .
$$

Proof. Equation (1.5) is equivalent to the higher order difference equation 2.12). Therefore, equation $(1.5)$ is positive if and only if the matrices $-\widehat{A}_{m}^{-1} \widehat{A}_{m-1}, \ldots$, $-\widehat{A}_{m}^{-1} \widehat{A}_{0}$ are positive, or equivalently, $\widehat{A}_{m}^{-1} \widehat{A}_{m-1}, \ldots, \widehat{A}_{m}^{-1} \widehat{A}_{0} \in \mathbb{R}_{-}^{n, n}$, see e.g. [10]. Similarly, if 1.5 is positive and asymptotically stable, then system 2.12 is positive and asymptotically stable, and therefore, see e.g. [17, this implies that

$$
\rho\left(-\widehat{A}_{m}^{-1} \widehat{A}_{m-1}-\ldots-\widehat{A}_{m}^{-1} \widehat{A}_{0}\right)<1 .
$$

The results in this section show that the asymptotic stability of a linear implicit difference equation can be characterized by the spectral properties of the matrix polynomial $P(\lambda)$. In the next section we use these results to compute stability radii.

3. Stability radii under restricted perturbations. Using the results from the previous section we can compute the eigenvalues of the matrix polynomial $P(\lambda)$ to characterize asymptotic stability of (1.1). Typically, however, the coefficient functions are not exactly known. Thus, a more realistic scenario for the stability analysis is to analyze the robustness of the asymptotic stability under small perturbations. To perform this analysis, in this section we study the behavior of the spectra when the coefficient matrices $\left(A_{m}, A_{m-1}, \ldots, A_{0}\right)$ under structured perturbations (see e.g. [28, 29]).

Consider a perturbed equation 1.5

$$
\widetilde{A}_{m} x^{k+m}+\widetilde{A}_{m-1} x^{k+m-1}+\ldots+\widetilde{A}_{0} x^{k}=0,
$$

with restricted perturbations of the form

$$
\left[\widetilde{A}_{m}, \widetilde{A}_{m-1}, \ldots, \widetilde{A}_{0}\right]=\left[A_{m}, A_{m-1} \ldots, A_{0}\right]+D \Delta E,
$$


where $D \in \mathbb{C}^{n, l}, E \in \mathbb{C}^{q, n(m+1)}$ are given structure matrices and $\Delta \in \mathbb{C}^{l, q}$ is the perturbation matrix. Using the abbreviation $\underline{A}=\left[A_{m}, A_{m-1}, \ldots, A_{0}\right]$ and introducing the set

$$
\Delta_{\mathbb{K}}=\left\{\Delta \in \mathbb{K}^{l, q} \mid \sqrt{3.1]} \text { is either singular or not asymptotically stable }\right\},
$$

where $\mathbb{K}=\mathbb{R}$ or $\mathbb{K}=\mathbb{C}$, we have the following definition.

Definition 3.1. Suppose that system (1.5) is asymptotically stable and let $\|\cdot\|$ be an operator norm on $\mathbb{C}^{l, q}$ that is induced by a vector norm. Then the stability radius of (1.5) with respect to structured perturbations of the form (3.2) is defined via

$$
r_{\mathbb{K}}^{D, E}(\underline{A})=\left\{\|\Delta\| \mid \Delta \in \Delta_{\mathbb{K}}\right\} .
$$

If $\Delta_{\mathbb{K}}=\emptyset$ then we set $r_{\mathbb{K}}^{D, E}(\underline{A})=\infty$. Define

$$
L(s):=\left[\begin{array}{c}
s^{m} I_{n} \\
s^{m-1} I_{n} \\
\vdots \\
I_{n}
\end{array}\right], E(s):=E L(s),
$$

and the transfer function $G(s)=E(s) P(s)^{-1} D$. In the following we will make use of the notion of structured distance to singularity of a nonsingular matrix $B \in \mathbb{C}^{n, n}$. Suppose that $D \in \mathbb{C}^{n, l}$ and $E \in \mathbb{C}^{q, n}$ are given structure matrices and $\|\cdot\|$ is an operator norm induced by a vector norm, then this distance is defined by

$$
d_{\mathbb{C}}^{D, E}(B)=\inf \left\{\|\Delta\| \mid \Delta \in \mathbb{C}^{l, q} \text { such that } B+D \Delta E \text { is singular }\right\} .
$$

It has been shown in [27] that the structured distance of $B$ to singularity is given by the formula

$$
d_{\mathbb{C}}^{D, E}(B)=\frac{1}{\left\|E B^{-1} D\right\|} .
$$

We have the following explicit formula for the complex structure stability radius.

THEOREM 3.2. Suppose that system 1.5 is asymptotically stable and subjected to structured perturbations of the form (3.2). Then the complex stability radius of 1.5. is given by

$$
r_{\mathbb{C}}^{D, E}(\underline{A})=\frac{1}{\sup _{|s| \in\{1, \infty\}}\|G(s)\|} .
$$

Proof. If the perturbed equation (3.1) is singular or it is regular but not asymptotically stable for $\Delta \in \mathbb{C}^{l, q}$, then this means that $\operatorname{det}\left(\widetilde{P}\left(s_{0}\right)\right)=0$ for some $s_{0} \in \mathbb{C} \backslash \mathbb{S}_{1}$, where $\widetilde{P}\left(s_{0}\right)=\widetilde{A}_{m} s_{0}^{m}+\widetilde{A}_{m-1} s_{0}^{m-1}+\ldots+\widetilde{A}_{0}$. By Definition 3.4, we obtain

$$
\begin{aligned}
\widetilde{P}\left(s_{0}\right) & =\left[\widetilde{A}_{m}, \widetilde{A}_{m-1}, \ldots, \widetilde{A}_{0}\right] L\left(s_{0}\right) \\
& =\left(\left[A_{m}, A_{m-1}, \ldots, A_{0}\right]+D \Delta E\right) L\left(s_{0}\right) \\
& =\left[A_{m}, A_{m-1}, \ldots, A_{0}\right] H\left(s_{0}\right)+D \Delta E L\left(s_{0}\right) \\
& =P\left(s_{0}\right)+D \Delta E\left(s_{0}\right) .
\end{aligned}
$$


Since system 1.5 is asymptotically stable, it follows that $P\left(s_{0}\right)$ is invertible. Hence, using the structured distance of $P\left(s_{0}\right)$ to singularity, we get

$$
\|\Delta\| \geq d_{\mathbb{C}}^{D, E\left(s_{0}\right)}\left(P\left(s_{0}\right)\right)=\frac{1}{\left\|E\left(s_{0}\right) P\left(s_{0}\right)^{-1} D\right\|}=\frac{1}{\left\|G\left(s_{0}\right)\right\|} \geq \frac{1}{\sup _{s \in \mathbb{C} \backslash \mathbb{S}_{1}}\|G(s)\|} .
$$

Since this inequality holds for any disturbance matrix $\Delta \in \mathbb{C}^{l, q}$ such that $D \Delta E$ destroys regularity or asymptotic stability of 1.5 , we obtain

$$
r_{\mathbb{C}}^{D, E}(\underline{A}) \geq \frac{1}{\sup _{s \in \mathbb{C} \backslash \mathbb{S}_{1}}\|G(s)\|} .
$$

To prove the converse inequality, we consider first the case that

$$
0<\sup _{s \in \mathbb{C} \backslash \mathbb{S}_{1}}\|G(s)\|<\infty .
$$

For any small $\epsilon>0$ such that $\sup _{s \in \mathbb{C} \backslash \mathbb{S}_{1}}\|G(s)\|-2 \epsilon>0$, there exists $s_{\epsilon} \in \mathbb{C} \backslash \mathbb{S}_{1}$ such that

$$
\left\|E\left(s_{\epsilon}\right) P\left(s_{\epsilon}\right)^{-1} D\right\|-\epsilon=\left\|G\left(s_{\epsilon}\right)\right\|-\epsilon \geq \sup _{s \in \mathbb{C} \backslash \mathbb{S}_{1}}\|G(s)\|-2 \epsilon .
$$

Using the structured distance to singularity, it follows that there exists a perturbation $\Delta_{\epsilon}$ such that

$$
\left\|\Delta_{\epsilon}\right\| \leq \frac{1}{\left\|E\left(s_{\epsilon}\right) P\left(s_{\epsilon}\right)^{-1} D\right\|-\epsilon}
$$

and the perturbed matrix $\widetilde{P}\left(s_{\epsilon}\right)=P\left(s_{\epsilon}\right)+D \Delta_{\epsilon} E\left(s_{\epsilon}\right)$ is not invertible. Hence, system 3.1 is not asymptotically stable when the perturbation $\Delta_{\epsilon}$ is applied, and thus,

$$
r_{\mathbb{C}}^{D, E}(\underline{A}) \leq\left\|\Delta_{\epsilon}\right\| \leq \frac{1}{\left\|G\left(s_{\epsilon}\right)\right\|-\epsilon} \leq \frac{1}{\sup _{s \in \mathbb{C} \backslash \mathbb{S}_{1}}\|G(s)\|-2 \epsilon} .
$$

Letting $\epsilon \rightarrow 0$, we get the required converse inequality. If $\sup _{s \in \mathbb{C} \backslash \mathbb{S}_{1}}\|G(s)\|=0$ then the converse inequality holds trivially, thus it remains to consider the final case that $\sup _{s \in \mathbb{C} \backslash \mathbb{S}_{1}}\|G(s)\|=\infty$. In this case, there exists a sequence $\left\{s_{n}\right\} \subset \mathbb{C} \backslash \mathbb{S}_{1}$ such that $\lim _{n \rightarrow \infty}\left\|G\left(s_{n}\right)\right\|=\infty$ and a sequence of perturbations $\left\{\Delta_{n}\right\}$ destroying the asymptotic stability of 1.5 such that

$$
r_{\mathbb{C}}^{D, E}(\underline{A}) \leq\left\|\Delta_{n}\right\| \leq \frac{2}{\left\|G\left(s_{n}\right)\right\|} .
$$

Letting $n \rightarrow \infty$, we get the converse inequality. Thus, we obtain

$$
r_{\mathbb{C}}^{D, E}(\underline{A})=\frac{1}{\sup _{s \in \mathbb{C} \backslash \mathbb{S}_{1}}\|G(s)\|} .
$$

Note that the function $G(s)=E(s) P(s)^{-1} D$ is analytic on $\mathbb{C} \backslash \mathbb{S}_{1}$. By the maximum principle, 20], $\|G(\cdot)\|$ either reaches its maximum value on the boundary $\partial \bar{S}_{1}$ or $\sup _{s \in \mathbb{C} \backslash \mathbb{S}_{1}}\|G(s)\|=\lim _{s \rightarrow \infty}\|G(s)\|$. Thus, we obtain

$$
r_{\mathbb{C}}^{D, E}(\underline{A})=\frac{1}{\sup _{|s| \in\{1, \infty\}}\|G(s)\|},
$$


and the proof is complete.

REMARK 3.3. Formula (3.6) for the stability radius of 1.5 is different from the formula for the stability radius of explicit difference equations as in [17, 25]. The reason is that we have to consider also the case that the function $\|G(s)\|$ obtains its supremum at infinity.

Unlike for the complex stability radius, a general formula for the real stability radius measured by an arbitrary matrix norm is not available. However, if we consider as vector norm the Euclidean norm, then a computable formula for the real stability radius can be established. For a matrix $M \in \mathbb{C}^{q, l}$, the real structured singular value of $M$ is defined by

$$
\mu_{\mathbb{R}}(M):=\left(\inf \left\{\|\Delta\|_{2} \mid \Delta \in \mathbb{R}^{l, q} \text {, and } \operatorname{det}\left(I_{l}+\Delta M\right)=0\right\}\right)^{-1},
$$

and it has been shown in [26] that the real structured singular value of $M \in \mathbb{C}^{q, l}$ is given by

$$
\mu_{\mathbb{R}}(M)=\inf _{\gamma \in(0,1]} \sigma_{2}\left[\begin{array}{cc}
\operatorname{Re} M & -\gamma \operatorname{Im} M \\
\frac{1}{\gamma} \operatorname{Im} M & \operatorname{Re} M
\end{array}\right]
$$

where $\sigma_{2}(H)$ denotes the second largest singular value of the matrix $H$.

Using this result, we obtain a formula for the real stability radius.

THEOREM 3.4. Suppose that 1.5 is asymptotically stable and subjected to structured perturbations of the form (3.2). Then the real stability radius of (1.5) (with respect to the Euclidean norm) is given by the formula

$$
r_{\mathbb{R}}^{D, E}(\underline{A})=\left(\sup _{s \in \mathbb{C} \backslash \mathbb{S}_{1}} \inf _{\gamma \in(0,1]} \sigma_{2}\left[\begin{array}{cc}
\operatorname{Re} G(s) & -\gamma \operatorname{Im} G(s) \\
\frac{1}{\gamma} \operatorname{Im} G(s) & \operatorname{Re} G(s)
\end{array}\right]\right)^{-1} .
$$

Proof. Suppose that the perturbed system $(3.1)$ is singular or it is regular but not asymptotically stable for a given $\Delta \in \mathbb{R}^{l, q}$. This means that

$$
\operatorname{det}\left(P\left(s_{0}\right)+D \Delta E\left(s_{0}\right)\right)=\operatorname{det}\left(\widetilde{P}\left(s_{0}\right)\right)=0
$$

for some $s_{0} \in \mathbb{C} \backslash \mathbb{S}_{1}$, and thus $\operatorname{det}\left(I_{n}+P\left(s_{0}\right)^{-1} D \Delta E\left(s_{0}\right)\right)=0$. Since for two matrices $B \in \mathbb{C}^{n, l}, C \in \mathbb{C}^{l \times n}$ one has $\operatorname{det}\left(I_{n}+B C\right)=0$ if and only if $\operatorname{det}\left(I_{l}+C B\right)=0$, this identity is equivalent to

$$
\operatorname{det}\left(I_{l}+\Delta E\left(s_{0}\right) P\left(s_{0}\right)^{-1} D\right)=\operatorname{det}\left(I_{l}+\Delta G\left(s_{0}\right)\right)=0 .
$$

The remainder of the proof is analogous to that of Theorem 3.2 and it follows that

$$
r_{\mathbb{R}}^{D, E}(\underline{A})=\frac{1}{\sup _{s \in \mathbb{C} \backslash \mathbb{S}_{1}} \mu_{\mathbb{R}}(G(s))},
$$

and by 3.8 , we obtain formula 3.9 .

REMARK 3.5. In formula 3.9 , we must take the supremum on $\mathbb{C} \backslash \mathbb{S}_{1}$ because the function $\mu_{\mathbb{R}}(G(s))$ may be discontinuous in $s$ for those $s$ for which $G(s)$ is a real matrix, see [26].

From the definition, it is easy to see that $r_{\mathbb{C}}^{D, E}(\underline{A}) \leq r_{\mathbb{R}}^{D, E}(\underline{A})$. Therefore, it is a natural question to study when the real and complex stability radii are equal. For linear explicit difference equations, it is known that these radii are equal if the 
system is positive and the structure matrices $D, E$ are positive, see [15, 16, 17]. In the following we will study this question for linear implicit difference equations. We need the following proposition which follows from the construction of a rank-one perturbation destroying the nonsingularity, see [17] or [27].

Proposition 3.6. Consider system (1.5) and suppose that $P(s)$ is nonsingular and $G(s)$ is a real matrix for some $s$. Then there exists a real perturbation $\Delta \in \mathbb{R}^{l, q}$ such that $\|\Delta\|=\|G(s)\|^{-1}$ and $P(s)+D \Delta E(s)$ is singular.

For $D$ in 3.2 and $\widehat{A}_{0}$ in $(2.2)$, we carry out the following tansformations and partitions

$$
W^{-1} D=\left[\begin{array}{c}
D^{(0)} \\
D^{(1)} \\
\vdots \\
D^{(m)}
\end{array}\right], \widehat{A}_{m}^{-1}=\left[M_{m}^{(0)}, M_{m}^{(1)}, \ldots, M_{m}^{(m)}\right]
$$

with $D^{(i)} \in \mathbb{C}^{d_{i}, l}, M_{m}^{(i)} \in \mathbb{C}^{n, d_{i}}$ for all $i=0, \ldots, m$. Introducing the matrix function $F(s):=\sum_{i=0}^{m} M_{m}^{(i)} D^{(i)} s^{i}$ and defining

$$
Q(s):=I_{n} s^{m}+\widehat{A}_{m}^{-1} \widehat{A}_{m-1} s^{m-1}+\ldots+\widehat{A}_{m}^{-1} \widehat{A}_{0}, I(s):=\left[\begin{array}{cccc}
I_{d_{0}} & 0 & \ldots & 0 \\
0 & s I_{d_{1}} & \ldots & 0 \\
\vdots & \vdots & \ddots & \vdots \\
0 & 0 & \ldots & s^{m} I_{d_{m}}
\end{array}\right] \text {, }
$$

we have the following result.

TheOREM 3.7. Suppose that 1.5 is strangeness-free, positive, and asymptotically stable and subjected to structured perturbations of the form (3.2) with $E \geq 0$ and $M_{m}^{(i)} D^{(i)} \geq 0$ for all $i=0, \ldots, m$. Then

$$
r_{\mathbb{C}}^{D, E}(\underline{A})=r_{\mathbb{R}}^{D, E}(\underline{A})=\frac{1}{\max \{\|G(1)\|,\|G(\infty)\|\}} .
$$

Proof. We only need to prove the converse inequality $r_{\mathbb{C}}^{D, E}(\underline{A}) \geq r_{\mathbb{R}}^{D, E}(\underline{A})$. Consider first the case that $\sup _{|s| \in\{1, \infty\}}\|G(s)\|=\sup _{|s|=1}\|G(s)\|$. Then we have

$$
\begin{aligned}
W^{-1} P(s) & =W^{-1} A_{m} s^{m}+W^{-1} A_{m-1} s^{m-1}+\ldots+W^{-1} A_{0} \\
& =\left[\begin{array}{c}
A_{m}^{(0)} \\
0 \\
0 \\
\vdots \\
0
\end{array}\right] s^{m}+\left[\begin{array}{c}
A_{m-1}^{(0)} \\
A_{m-1}^{(1)} \\
0 \\
\vdots \\
0
\end{array}\right] s^{m-1}+\ldots+\left[\begin{array}{c}
A_{0}^{(0)} \\
A_{0}^{(1)} \\
A_{0}^{(2)} \\
\vdots \\
A_{0}^{(m)}
\end{array}\right] .
\end{aligned}
$$

Using (2.2) and (3.11) it follows that

$$
I(s) W^{-1} P(s)=\widehat{A}_{m} s^{m}+\widehat{A}_{m-1} s^{m-1}+\ldots+\widehat{A}_{0}=\widehat{A}_{m} Q(s),
$$

and hence,

$$
\begin{aligned}
P(s) & =W I(s)^{-1} \widehat{A}_{m} Q(s), \\
P(s)^{-1} & =Q(s)^{-1} \widehat{A}_{m}^{-1} I(s) W^{-1}, \\
G(s) & =E(s) Q(s)^{-1} \widehat{A}_{m}^{-1} I(s) W^{-1} D .
\end{aligned}
$$


Using (3.10) we have

$$
\widehat{A}_{m}^{-1} I(s) W^{-1} D=\sum_{i=0}^{m} M_{m}^{(i)} D^{(i)} s^{i}=F(s),
$$

and hence $G(s)=E(s) Q(s)^{-1} F(s)$. Since the system 1.5 is strangeness-free and positive, it follows that the matrices $\widehat{A}_{m}^{-1} \widehat{A}_{m-1}, \ldots, \widehat{A}_{m}^{-1} \widehat{A}_{0}$ are nonpositive. Therefore, for all $z \in \mathbb{C}$ with $|z|=1$, we have

$$
\left|-\widehat{A}_{m}^{-1} \widehat{A}_{m-1} z-\ldots-\widehat{A}_{m}^{-1} \widehat{A}_{0} z^{m}\right| \leq-\widehat{A}_{m}^{-1} \widehat{A}_{m-1}-\ldots-\widehat{A}_{m}^{-1} \widehat{A}_{0} .
$$

and thus, by Proposition 2.8 for these $z$ we have

$$
\rho\left(-\widehat{A}_{m}^{-1} \widehat{A}_{m-1} z-\ldots-\widehat{A}_{m}^{-1} \widehat{A}_{0} z^{m}\right)<1 .
$$

Hence, for $s \in \mathbb{C}$ with $|s|=1$, and $z=1 / s$ we have

$$
\begin{aligned}
\left|Q(s)^{-1}\right| & =\left|\left(I_{n}-\left(-\widehat{A}_{m}^{-1} \widehat{A}_{m-1} z-\ldots-\widehat{A}_{m}^{-1} \widehat{A}_{0} z^{m}\right)\right)^{-1}\right| \\
& =\left|\sum_{i=0}^{\infty}\left(-\widehat{A}_{m}^{-1} \widehat{A}_{m-1} z-\ldots-\widehat{A}_{m}^{-1} \widehat{A}_{0} z^{m}\right)^{i}\right| \\
& \leq \sum_{i=0}^{\infty}\left|-\widehat{A}_{m}^{-1} \widehat{A}_{m-1} z-\ldots-\widehat{A}_{m}^{-1} \widehat{A}_{0} z^{m}\right|^{i} \\
& \leq \sum_{i=0}^{\infty}\left(-\widehat{A}_{m}^{-1} \widehat{A}_{m-1}-\ldots-\widehat{A}_{m}^{-1} \widehat{A}_{0}\right)^{i} \\
& =\left(I_{n}-\left(-\widehat{A}_{m}^{-1} \widehat{A}_{m-1}-\ldots-\widehat{A}_{m}^{-1} \widehat{A}_{0}\right)\right)^{-1}=Q(1)^{-1} .
\end{aligned}
$$

Moreover, since $|s|=1$, we have

$$
\begin{aligned}
& |E(s)|=|E H(s)|=E|H(s)| \leq E H(|s|)=E H(1)=E(1), \\
& |F(s)|=\left|\sum_{i=0}^{m} M_{m}^{(i)} D^{(i)} s^{i}\right| \leq \sum_{i=0}^{m}\left|M_{m}^{(i)} D^{(i)}\right||s|^{i}=\sum_{i=0}^{m} M_{m}^{(i)} D^{(i)}=F(1),
\end{aligned}
$$

and thus we get

$$
|G(s)| \leq|E(s)|\left|Q(s)^{-1}\right||F(s)| \leq E(1) Q(1)^{-1} F(1)=G(1) .
$$

Since $G(1)$ is a real matrix, by Proposition 3.6 , there exist a real destablizing perturbation $\Delta$ such that

$$
\|\Delta\|=\frac{1}{\|G(1)\|}=\frac{1}{\sup _{|s|=1}\|G(s)\|}=r_{\mathbb{C}}^{D, E}(\underline{A}),
$$

which implies that $r_{\mathbb{R}}^{D, E}(\underline{A}) \leq\|\Delta\|=r_{\mathbb{C}}^{D, E}(\underline{A})$, and hence in this case

$$
r_{\mathbb{C}}^{D, E}(\underline{A})=r_{\mathbb{R}}^{D, E}(\underline{A})=\frac{1}{\|G(1)\|} .
$$

For the second case that $\sup _{|s| \in\{1, \infty\}}\|G(s)\|=\|G(\infty)\|=\lim _{s \rightarrow \infty}\|G(s)\|$, it follows that

$$
r_{\mathbb{C}}^{D, E}(\underline{A})=\lim _{n \rightarrow \infty} \frac{1}{\|G(n)\|} .
$$


Since $G(n)=E(n) Q(n)^{-1} F(n)$ is a real matrix, by Proposition 3.6 there exists a real destablizing perturbation $\Delta_{n}$ such that $\left\|\Delta_{n}\right\|=\frac{1}{\|G(n)\|}$ and this implies that

$$
r_{\mathbb{R}}^{D, E}(\underline{A}) \leq \lim _{n \rightarrow \infty} \frac{1}{\|G(n)\|}=r_{\mathbb{C}}^{D, E}(\underline{A}) .
$$

Thus, in this case, we obtain

$$
r_{\mathbb{C}}^{D, E}(\underline{A})=r_{\mathbb{R}}^{D, E}(\underline{A})=\frac{1}{\|G(\infty)\|},
$$

and the proof is complete.

We illustrate our results with the following example.

EXAMPLE 3.8. Consider the second order implicit difference equation $A_{2} x^{k+2}+$ $A_{1} x^{k+1}+A_{0} x^{k}=0$, with

$$
A_{2}=\left[\begin{array}{ll}
2 & 0 \\
0 & 0
\end{array}\right], A_{1}=\left[\begin{array}{ll}
0 & 0 \\
0 & 2
\end{array}\right], A_{0}=\left[\begin{array}{cc}
-1 & 0 \\
0 & -1
\end{array}\right]
$$

and suppose that the structurally perturbed system is of the form

$$
\left[\widetilde{A}_{2}, \widetilde{A}_{1}, \widetilde{A}_{0}\right]=\left[\begin{array}{cccccc}
2+\delta_{1} & 0 & \delta_{2} & \delta_{2} & -1+\delta_{2} & 0 \\
\delta_{1} & 0 & \delta_{2} & 2+\delta_{2} & \delta_{2} & -1
\end{array}\right]=\left[A_{2}, A_{1}, A_{0}\right]+D \Delta E,
$$

with

$$
D=\left[\begin{array}{l}
1 \\
1
\end{array}\right], E=\left[\begin{array}{cccccc}
1 & 0 & 0 & 0 & 0 & 0 \\
0 & 0 & 1 & 1 & 1 & 0
\end{array}\right], \Delta=\left[\begin{array}{ll}
\delta_{1} & \delta_{2}
\end{array}\right]
$$

and disturbance parameters $\delta_{1}, \delta_{2} \in \mathbb{C}$.

The unperturbed system is strangeness-free and positive and has the transformed matrices

$$
\widehat{A}_{2}=\left[\begin{array}{ll}
2 & 0 \\
0 & 2
\end{array}\right], \widehat{A}_{1}=\left[\begin{array}{cc}
0 & 0 \\
0 & -1
\end{array}\right], \widehat{A}_{0}=\left[\begin{array}{cc}
-1 & 0 \\
0 & 0
\end{array}\right] .
$$

The spectrum of the matrix polynomial

$$
P(s)=\left[\begin{array}{cc}
2 s^{2}-1 & 0 \\
0 & 2 s-1
\end{array}\right]
$$

satisfies $\sigma(P) \subset \mathbb{S}_{1}$ and thus this system is asymptotically stable. We have $E \geq$ $0, M_{2}^{(0)} D^{(0)}=M_{2}^{(1)} D^{(1)}=1 / 2>0$, and $M_{2}^{(2)} D^{(2)}=0$, and by simple algebraic manipulations we obtain

$$
G(s)=E(s) P(s)^{-1} D=\left[\begin{array}{cc}
s^{2} & 0 \\
s+1 & s
\end{array}\right]\left[\begin{array}{cc}
\frac{1}{2 s^{2}-1} & 0 \\
0 & \frac{1}{2 s-1}
\end{array}\right]\left[\begin{array}{l}
1 \\
1
\end{array}\right]=\left[\begin{array}{c}
\frac{s^{2}}{2 s^{2}-1} \\
\frac{s+1}{2 s^{2}-1}+\frac{s}{2 s-1}
\end{array}\right] .
$$

It follows that $G(1)=\left[\begin{array}{ll}1 & 3\end{array}\right]^{T}$ and $G(\infty)=\left[\begin{array}{ll}1 / 2 & 1 / 2\end{array}\right]^{T}$. Then, by Theorem 3.7 . with respect to the maximum norm, we obtain

$$
r_{\mathbb{C}}^{D, E}(\underline{A})=r_{\mathbb{R}}^{D, E}(\underline{A})=\frac{1}{\max \{\|G(1)\|,\|G(\infty)\|\}}=\frac{1}{\max \{3, \sqrt{1 / 2}\}}=\frac{1}{3} .
$$


We can also apply the discussed results for the special case of blockwise perturbations of the form

$$
\widetilde{A}_{i}=A_{i}+D \Delta_{i} E_{i}, i=0, \ldots, m
$$

with $\Delta_{i} \in \mathbb{C}^{l, q_{i}}, E_{i} \in \mathbb{C}^{q_{i}, n}, i=0,1, \ldots, m$, and $D \in \mathbb{C}^{n, l}$. The perturbed system 3.13 can be represented in the form

$$
\left[A_{m}, A_{m-1}, \ldots, A_{0}\right]+D \Delta E
$$

with $E=\operatorname{diag}\left(E_{m}, E_{m-1}, \ldots, E_{0}\right)$ and $\Delta=\left[\Delta_{m}, \Delta_{m-1}, \ldots, \Delta_{0}\right]$. Applying Theorems 3.2 and Theorem 3.4 we get the stability radii $r_{\mathbb{C}}^{D, E}(\underline{A}), r_{\mathbb{R}}^{D, E}(\underline{A})$ of equation 1.5 under these structured perturbations. As a special case, we consider equation 1.5 with perturbed coefficients

$$
\widetilde{A}_{i}=A_{i}+\alpha_{i} \Delta_{i}, i=0, \ldots, m
$$

with $\alpha_{i} \in \mathbb{C}, \Delta_{i} \in \mathbb{C}^{n, n}, i=0, \ldots, m$. Denoting by $r_{\mathbb{C}}^{\alpha}(\underline{A})$ the stability radius of equation (1.5) under the perturbations (3.14) and setting

$$
\xi(s)=\left(\sum_{i=0}^{m}\left|\alpha_{i}\right|^{p}|s|^{i p}\right)^{1 / p},
$$

we have the following proposition.

Proposition 3.9. Suppose that equation 1.5 is asymptotically stable and subjected to perturbations of the form (3.14). If we consider as norm the p-norm with $0<p<\infty$, then

$$
r_{\mathbb{C}}^{\alpha}(\underline{A})=\min \left\{\frac{1}{\xi(1) \max _{|s|=1}\left\|P(s)^{-1}\right\|}, \frac{1}{\lim _{s \rightarrow \infty} \xi(s)\left\|P(s)^{-1}\right\|}\right\} .
$$

Proof. In this case we have $D=I_{n}$ and $E_{i}=\alpha_{i} I_{n}, i=0, \ldots, m$, i.e.

$$
E=\operatorname{diag}\left(\alpha_{m} I_{n}, \alpha_{m-1} I_{n}, \ldots, \alpha_{0} I_{n}\right), E(s)=\left[\begin{array}{c}
\alpha_{m} s^{m} I_{n} \\
\alpha_{m-1} s^{m-1} I_{n} \\
\vdots \\
\alpha_{0} I_{n}
\end{array}\right], G(s)=E(s) P(s)^{-1} .
$$

It is easy to see that for $v \in \mathbb{C}^{n}$, we have $\|E(s) v\|_{p}=\xi(s)\|v\|_{p}$ and this implies that $\|G(s)\|=\xi(s)\left\|P(s)^{-1}\right\|$. Note that $\xi(s)=\xi(1)$ for $s \in \mathbb{C}$ with $|s|=1$. Thus, using Theorem 3.2 the assertion follows.

In this section we have shown that the classical results of [15] on stability radii for homogeneous systems can be extended to higher order difference equations with singular leading coefficients. However, in the inhomogeneous case, there are restrictions to the initial conditions in terms of the inhomogeneity. In particular, it may happen that due to the perturbation the index changes and thus for nonhomogeneous systems and nonzero initial conditions the solvability may be destroyed. This topic is discussed in the next section. 
4. Index preserving perturbations. It is already known for the case of perturbed nonhomogeneous differential-algebraic equations (DAEs) [5], see also [8, 9], that it is necessary to restrict the perturbations in order to get a meaningful concept of the structured stability radius, since under infinitesimally small perturbations the solvability may be destroyed when the index changes, because then the consistency conditions for initial conditions and inhomogeneity may change drastically. This means that a regular system may become singular in this way. We therefore introduce the following set of admissible perturbations.

Suppose that system $(1.5)$ is asymptotically stable and consider a perturbed system

$$
\left(A_{m}+D_{m} \Delta_{m} E\right) x^{k+m}+\left(A_{m-1}+D_{m-1} \Delta_{m-1} E\right) x^{k+m-1}+\ldots+\left(A_{0}+D_{0} \Delta_{0} E\right) x^{k}=f^{k},
$$

where $\Delta_{i} \in \mathbb{C}^{l_{i}, q}, i=0, \ldots, m$ are perturbations and $D_{i} \in \mathbb{C}^{n, l_{i}}, E \in \mathbb{C}^{q, n}, i=$ $0, \ldots, m$ are matrices that restrict the structure of the perturbations.

Set

$$
\Delta=\left[\begin{array}{c}
\Delta_{m} \\
\Delta_{m-1} \\
\vdots \\
\Delta_{0}
\end{array}\right], D=\left[\begin{array}{llll}
D_{m} & D_{m-1} & \ldots & D_{0}
\end{array}\right]
$$

$l=l_{0}+l_{1}+\ldots+l_{m}$ and consider the set of destabilizing perturbations

$$
\mathcal{V}_{\mathbb{K}}=\left\{\Delta \in \mathbb{K}^{l, q} \mid 4.1 \text { is non-regular or not asymptotically stable }\right\} \text {. }
$$

Then we define the structured stability radius of 1.5 subject to structured perturbations as in (4.1) as

$$
r_{\mathbb{K}}(\underline{A} ; D, E)=\inf \left\{\|\Delta\|: \Delta \in \mathcal{V}_{\mathbb{K}}\right\},
$$

where $\|\cdot\|$ is again a matrix norm induced by a vector norm.

DeFINITION 4.1. Consider a strangeness-free system 1.5 and let $W \in \mathbb{C}^{n, n}$ be such that (2.10) holds. A structured perturbation as in (4.1) is called admissible if (4.1) is still strangeness-free with the same triple $\left(d_{0}, d_{1}, \ldots, d_{m}\right)$, i.e., there exists a nonsingular matrix $\tilde{W} \in \mathbb{C}^{n, n}$ such that

$$
\begin{aligned}
\tilde{W}^{-1}\left(A_{m}+D_{m} \Delta_{m} E\right) & =\left[\begin{array}{c}
\tilde{A}_{m}^{(0)} \\
0 \\
0 \\
\vdots \\
0
\end{array}\right], \tilde{W}^{-1}\left(A_{m-1}+D_{m-1} \Delta_{m-1} E\right)=\left[\begin{array}{c}
\tilde{A}_{m-1}^{(0)} \\
\tilde{A}_{m-1}^{(1)} \\
0 \\
\vdots \\
0
\end{array}\right] \\
\tilde{W}^{-1}\left(A_{0}+D_{0} \Delta_{0} E\right) & =\left[\begin{array}{c}
\tilde{A}_{0}^{(0)} \\
\tilde{A}_{0}^{(1)} \\
\tilde{A}_{0}^{(2)} \\
\vdots \\
\tilde{A}_{0}^{(m)}
\end{array}\right]
\end{aligned}
$$


where $\tilde{A}_{m}^{(0)}, \tilde{A}_{m-1}^{(0)}, \ldots, \tilde{A}_{0}^{(0)} \in \mathbb{C}^{d_{0}, n}, \tilde{A}_{m-1}^{(1)}, \ldots, \tilde{A}_{0}^{(1)} \in \mathbb{C}^{d_{1}, n}, \ldots \tilde{A}_{0}^{(m)} \in \mathbb{C}^{d_{m}, n}$, are such that

$$
\left[\begin{array}{c}
\tilde{A}_{m}^{(0)} \\
\tilde{A}_{m-1}^{(1)} \\
\vdots \\
\tilde{A}_{0}^{(m)}
\end{array}\right]
$$

is invertible.

Suppose that the matrices $D_{i}, i=0,1, \ldots, m$, that are restricting the structure have the form

$$
W^{-1} D_{m}=\left[\begin{array}{c}
D_{m}^{(0)} \\
D_{m}^{(1)} \\
\vdots \\
D_{m}^{(m)}
\end{array}\right], W^{-1} D_{m-1}=\left[\begin{array}{c}
D_{m-1}^{(0)} \\
D_{m-1}^{(1)} \\
\vdots \\
D_{m-1}^{(m)}
\end{array}\right], \ldots, W^{-1} D_{0}=\left[\begin{array}{c}
D_{0}^{(0)} \\
D_{0}^{(1)} \\
\vdots \\
D_{0}^{(m)}
\end{array}\right],
$$

where $D_{m}^{(j)} \in \mathbb{C}^{d_{j}, l_{m}}, D_{m-1}^{(j)} \in \mathbb{C}^{d_{j}, l_{m-1}}, \ldots, D_{0}^{(j)} \in \mathbb{C}^{d_{j}, l_{0}}, j=0,1, \ldots, m$. According to [5, Lemma 3.3], if the structured perturbation is admissible, then $D_{i}^{(j)} \Delta_{i} E=0$ for $0 \leq i, j \leq m$ such that $i+j>m$. This can be achieved by requiring that

$$
D_{i}^{(j)}=0,0 \leq i, j \leq m, i+j>m .
$$

Note that by Remark 2.6, condition 4.6 is invariant with respect to the choice of the transformation matrix $W$. Furthermore, it is easy to see that for structured perturbations satisfying (4.6), if the perturbation $\Delta$ is sufficiently small, then the strangeness-free property is preserved with the same sizes of the blocks.

We denote the infimum of the norm of all perturbations $\Delta$ such that 4.1 is no longer strangeness-free or for which the sizes of the blocks $d_{0}, d_{1}, \ldots, d_{m}$ change by $d_{\mathbb{C}}^{s}(\underline{A} ; D, E)$, and immediately have the following proposition.

Proposition 4.2. Suppose that equation (1.5) is strangeness-free and subjected to structured perturbations with $D_{i}, i=0,1, \ldots, m$ satisfying (4.6). Then

$$
d_{\mathbb{C}}^{s}(\underline{A} ; D, E)=\left\|E\left[\begin{array}{c}
A_{m}^{(0)} \\
A_{m-1}^{(1)} \\
\vdots \\
A_{0}^{(m)}
\end{array}\right]^{-1}\left[\begin{array}{cccc}
D_{m}^{(0)} & 0 & \cdots & 0 \\
0 & D_{m-1}^{(1)} & \ddots & \vdots \\
\vdots & \ddots & \ddots & 0 \\
0 & \cdots & 0 & D_{0}^{(m)}
\end{array}\right]\right\|^{-1} .
$$

Proof. With restriction matrices $D_{i}, i=0,1, \ldots, m$ satisfying 4.6 , the perturbed system 4.1] is still strangeness-free with $\tilde{A}_{j}^{(0)} \in \mathbb{C}^{d_{0}, n}, j=0, \ldots, m, \tilde{A}_{j}^{(1)} \in \mathbb{C}^{d_{1}, n}$, $j=0, \ldots, m-1, \ldots, \tilde{A}_{0}^{(m)} \in \mathbb{C}^{d_{m}, n}$ as in 4.4, if and only if

$$
\left[\begin{array}{c}
A_{m}^{(0)}+D_{m}^{(0)} \Delta_{m} E \\
A_{m-1}^{(1)}+D_{m-1}^{(1)} \Delta_{m-1} E \\
\vdots \\
A_{0}^{(m)}+D_{0}^{(m)} \Delta_{0} E
\end{array}\right]=\left[\begin{array}{c}
A_{m}^{(0)} \\
A_{m-1}^{(1)} \\
\vdots \\
A_{0}^{(m)}
\end{array}\right]+\left[\begin{array}{cccc}
D_{m}^{(0)} & 0 & \cdots & 0 \\
0 & D_{m-1}^{(1)} & \ddots & \vdots \\
\vdots & \ddots & \ddots & 0 \\
0 & \cdots & 0 & D_{0}^{(m)}
\end{array}\right] \Delta E
$$


is nonsingular. Thus employing again the distance of a nonsingular matrix to the nearest singular matrix, we obtain

$$
d_{\mathbb{C}}^{s}(\underline{A} ; D, E)=\left\|E\left[\begin{array}{c}
A_{m}^{(0)} \\
A_{m-1}^{(1)} \\
\vdots \\
A_{0}^{(m)}
\end{array}\right]^{-1}\left[\begin{array}{cccc}
D_{m}^{(0)} & 0 & \cdots & 0 \\
0 & D_{m-1}^{(1)} & \ddots & \vdots \\
\vdots & \ddots & \ddots & 0 \\
0 & \cdots & 0 & D_{0}^{(m)}
\end{array}\right]\right\|^{-1} .
$$

Remark 4.3. Again, by Remark 2.6, it is not difficult to show that in fact the formula in Proposition 4.2 is independent of the choice of the transformation matrix $W$.

Defining

$$
D(s):=\left[\begin{array}{llll}
s^{m} D_{m} & s^{m-1} D_{m-1} & \ldots & D_{0}
\end{array}\right], H(s):=E P(s)^{-1} D(s)
$$

we have the following proposition.

Proposition 4.4. Consider an asymptotically stable system of the form (1.5). If the system is strangeness-free and subjected to structured perturbations as in (4.1) with structure matrices $D_{i}$ satisfying 4.6 and if the perturbation $\Delta$ satisfies

$$
\|\Delta\|<\|H(\infty)\|^{-1}=\left(\lim _{s \rightarrow \infty}\|H(s)\|\right)^{-1},
$$

then the structured perturbation is admissible, i.e., the perturbed equation (4.1) is strangeness-free with the same block-sizes $d_{0}, d_{1}, \ldots, d_{m}$.

Proof. To prove the assertion, we will show that

$$
\left(\lim _{s \rightarrow \infty}\|H(s)\|\right)^{-1}=\left\|E\left[\begin{array}{c}
A_{m}^{(0)} \\
A_{m-1}^{(1)} \\
\vdots \\
A_{0}^{(m)}
\end{array}\right]^{-1}\left[\begin{array}{cccc}
D_{m}^{(0)} & 0 & \cdots & 0 \\
0 & D_{m-1}^{(1)} & \ddots & \vdots \\
\vdots & \ddots & \ddots & 0 \\
0 & \cdots & 0 & D_{0}^{(m)}
\end{array}\right]\right\|^{-1} .
$$

We can rewrite $H$ as

$$
\begin{aligned}
& H(s)=E P(s)^{-1}\left[\begin{array}{llll}
s^{m} D_{m} & s^{m-1} D_{m-1} & \ldots & D_{0}
\end{array}\right] \\
& =E\left[\begin{array}{c}
A_{m}^{(0)} s^{m}+A_{m-1}^{(0)} s^{m-1}+\ldots+A_{0}^{(0)} \\
A_{m-1}^{(1)} s^{m-1}+\ldots+A_{0}^{(1)} \\
\vdots \\
A_{0}^{(m)}
\end{array}\right]^{-1}\left[\begin{array}{cccc}
s^{m} D_{m}^{(0)} & s^{m-1} D_{m-1}^{(0)} & \ldots & D_{0}^{(0)} \\
0 & s^{m-1} D_{m-1}^{(1)} & \ldots & D_{0}^{(1)} \\
\vdots & \ddots & \ddots & \vdots \\
0 & \ldots & 0 & D_{0}^{(m)}
\end{array}\right] \\
& =: E \Psi(s) \text {, }
\end{aligned}
$$

and thus it follows that

$$
\left[\begin{array}{c}
A_{m}^{(0)} s^{m}+A_{m-1}^{(0)} s^{m-1}+\ldots+A_{0}^{(0)} \\
A_{m-1}^{(1)} s^{m-1}+\ldots+A_{0}^{(1)} \\
\vdots \\
A_{0}^{(m)}
\end{array}\right] \Psi(s)=\left[\begin{array}{cccc}
s^{m} D_{m}^{(0)} & s^{m-1} D_{m-1}^{(0)} & \ldots & D_{0}^{(0)} \\
0 & s^{m-1} D_{m-1}^{(1)} & \ldots & D_{0}^{(1)} \\
\vdots & \ddots & \ddots & \vdots \\
0 & \ldots & 0 & D_{0}^{(m)}
\end{array}\right]
$$


If $s \neq 0$, then this is equivalent to

$$
\left[\begin{array}{c}
A_{m}^{(0)}+A_{m-1}^{(0)} / s+\ldots+A_{0}^{(0)} / s^{m} \\
A_{m-1}^{(1)}+\ldots+A_{0}^{(1)} / s^{m-1} \\
\vdots \\
A_{0}^{(m)}
\end{array}\right] \Psi(s)=\left[\begin{array}{cccc}
D_{m}^{(0)} & D_{m-1}^{(0)} / s & \ldots & D_{0}^{(0)} / s^{m} \\
0 & D_{m-1}^{(1)} & \ldots & D_{0}^{(1)} / s^{m-1} \\
\vdots & \ddots & \ddots & \vdots \\
0 & \ldots & 0 & D_{0}^{(m)}
\end{array}\right]
$$

Since

$$
\lim _{s \rightarrow \infty}\left[\begin{array}{c}
A_{m}^{(0)}+A_{m-1}^{(0)} / s+\ldots+A_{0}^{(0)} / s^{m} \\
A_{m-1}^{(1)}+\ldots+A_{0}^{(1)} / s^{m-1} \\
\vdots \\
A_{0}^{(m)}
\end{array}\right]=\left[\begin{array}{c}
A_{m}^{(0)} \\
A_{m-1}^{(1)} \\
\vdots \\
A_{0}^{(m)}
\end{array}\right]
$$

and

$$
\lim _{s \rightarrow \infty}\left[\begin{array}{cccc}
D_{m}^{(0)} & D_{m-1}^{(0)} / s & \ldots & D_{0}^{(0)} / s^{m} \\
0 & D_{m-1}^{(1)} & \ldots & D_{0}^{(1)} / s^{m-1} \\
\vdots & \ddots & \ddots & \vdots \\
0 & \ldots & 0 & D_{0}^{(m)}
\end{array}\right]=\left[\begin{array}{cccc}
D_{m}^{(0)} & 0 & \ldots & 0 \\
0 & D_{m-1}^{(1)} & \ddots & \vdots \\
\vdots & \ddots & \ddots & 0 \\
0 & \ldots & 0 & D_{0}^{(m)}
\end{array}\right]
$$

it follows that

$$
\lim _{s \rightarrow \infty} \Psi(s)=\left[\begin{array}{c}
A_{m}^{(0)} \\
A_{m-1}^{(1)} \\
\vdots \\
A_{0}^{(m)}
\end{array}\right]^{-1}\left[\begin{array}{cccc}
D_{m}^{(0)} & 0 & \cdots & 0 \\
0 & D_{m-1}^{(1)} & \ddots & \vdots \\
\vdots & \ddots & \ddots & 0 \\
0 & \cdots & 0 & D_{0}^{(m)}
\end{array}\right]
$$

Thus, we have that

$$
H(\infty)=\lim _{s \rightarrow \infty} H(s)=E \lim _{s \rightarrow \infty} \Psi(s)=E\left[\begin{array}{c}
A_{m}^{(0)} \\
A_{m-1}^{(1)} \\
\vdots \\
A_{0}^{(m)}
\end{array}\right]^{-1}\left[\begin{array}{cccc}
D_{m}^{(0)} & 0 & \ldots & 0 \\
0 & D_{m-1}^{(1)} & \ddots & \vdots \\
\vdots & \ddots & \ddots & 0 \\
0 & \cdots & 0 & D_{0}^{(m)}
\end{array}\right],
$$

and hence 4.7 holds. By Proposition 4.2 it then follows that if

$$
\|\Delta\|<\|H(\infty)\|^{-1}
$$

then the perturbed equation (4.1) is strangeness-free with the same blocksizes $d_{0}, d_{1}$, $\ldots, d_{m}$ as for 1.5 .

We then have the following result characterizing the stability radius for strangenessfree implicit difference equations under admissible structured perturbations.

THEOREM 4.5. Suppose that equation (1.5) is asymptotically stable and strangeness-free and subjected to structured perturbations as in (4.1). Then

$$
r_{\mathbb{C}}(\underline{A} ; D, E)=\frac{1}{\sup _{|s| \in\{1, \infty\}}\|H(s)\|} .
$$


Furthermore, if the structure matrices $D_{i}$ satisfy (4.6) and $\|\Delta\|<r_{\mathbb{C}}(\underline{A} ; D, E)$, then (4.1) is strangeness-free with the same blocksizes $d_{0}, d_{1}, \ldots, d_{m}$ as for (1.5).

Proof. If $\widetilde{P}(s)$ is the characteristic polynomial of the perturbed equation 4.1, then we have

$$
\begin{aligned}
\widetilde{P}(s)= & \widetilde{A}_{m} s^{m}+\widetilde{A}_{m-1} s^{m-1}+\ldots+\widetilde{A}_{0} \\
= & \left(A_{m}+D_{m} \Delta_{m} E\right) s^{m}+\left(A_{m-1}+D_{m-1} \Delta_{m-1} E\right) s^{m-1}+\ldots+\left(A_{0}+D_{0} \Delta_{0} E\right) \\
= & \left(A_{m} s^{m}+A_{m-1} s^{m-1}+\ldots+A_{0}\right)+ \\
& \quad\left(s^{m} D_{m} \Delta_{m}+s^{m-1} D_{m-1} \Delta_{m-1}+\ldots+D_{0} \Delta_{0}\right) E \\
= & P(s)+D(s) \Delta E .
\end{aligned}
$$

Similar to the proof of Theorem 3.2 we obtain that

$$
r_{\mathbb{C}}(\underline{A} ; D, E)=\inf _{s \in \mathbb{C} \backslash \mathbb{S}_{1}} d_{\mathbb{C}}^{D(s), E}(P(s))=\frac{1}{\sup _{s \in \mathbb{C} \backslash \mathbb{S}_{1}}\|H(s)\|}=\frac{1}{\sup _{|s| \in\{1, \infty\}}\|H(s)\|} .
$$

By Proposition 4.4, we then have that 4.1 is strangeness-free if $\|\Delta\|<r_{\mathbb{C}}(\underline{A} ; D, E)$.

(

For the case of positive equations, we get the following result on the real stability radius.

Theorem 4.6. Let equation 1.5 be strangeness-free and positive. Assume that (1.5) is asymptotically stable and subjected to structured perturbations as in 4.1) with $E \geq 0$ and $M_{m}^{(i)} D_{j}^{(i)} \geq 0$ for all $i, j=0, \ldots, m$. Then we have

$$
r_{\mathbb{C}}(\underline{A} ; D, E)=r_{\mathbb{R}}(\underline{A} ; D, E)=\frac{1}{\max \{\|H(1)\|,\|H(\infty)\|\}} .
$$

Furthermore, if the structure matrices $D_{i}$ satisfy 4.6 and $\|\Delta\|<r_{\mathbb{C}}(\underline{A} ; D, E)$, then (4.1) is strangeness-free with the same blocksizes $d_{0}, d_{1}, \ldots, d_{m}$ as for (1.5).

Proof. The proof is analogous to that of Theorem 3.7. $\mathrm{c}$

As a final result we consider the case that equation (1.5) is subjected to blockwise structured perturbations of the form

$$
\widetilde{A}_{m} x^{k+m}+\widetilde{A}_{m-1} x^{k+m-1}+\ldots+\widetilde{A}_{0} x^{k}=0,
$$

with

$$
\widetilde{A}_{i}=A_{i}+D_{i} \Delta_{i} E_{i}
$$

where $D_{i} \in \mathbb{C}^{n, l_{i}}$, and $E_{i} \in \mathbb{C}^{q_{i}, n}, i=0, \ldots, m$ are given structure matrices and $\Delta_{i} \in \mathbb{C}^{l_{i}, q_{i}}, i=0, \ldots, m$ are unknown disturbance matrices.

These perturbation can be described as a blockdiagonal perturbation

$$
\underline{\widetilde{A}}=\underline{A}+D \Delta_{b} E,
$$

where $D=\left[D_{m}, D_{m-1}, \ldots, D_{0}\right], E=\operatorname{diag}\left(E_{m}, E_{m-1}, \ldots, E_{0}\right)$ and

$$
\Delta_{b}=\operatorname{diag}\left(\Delta_{m}, \Delta_{m-1}, \ldots, \Delta_{0}\right) .
$$

We endow the linear space of blockdiagonal perturbations with the norm

$$
\left\|\Delta_{b}\right\|=\sum_{i=0}^{m}\left\|\Delta_{i}\right\| .
$$


If equation 1.5 is asymptotically stable, then we define

$$
\Xi_{\mathbb{K}}=\left\{\Delta_{b} \mid \Delta_{i} \in \mathbb{K}^{l_{i}, q_{i}}, 4.9 \text { is non-regular or not asymptot. stable }\right\}
$$

and the stability radius of 1.5 with respect to perturbations of the form 4.10 is defined as

$$
r_{\mathbb{K}}^{b}(\underline{A})=\inf \left\{\left\|\Delta_{b}\right\| \mid \Delta_{b} \in \Xi_{\mathbb{K}}\right\} .
$$

For each tuple of functions $\left(\beta_{0}(s), \beta_{1}(s), \ldots, \beta_{m}(s)\right)$ such that $\beta_{i}(s) \neq 0$ for all $s \in$ $\mathbb{C} \backslash \mathbb{S}_{1}$ and $i, j=0, \ldots, m$, we define the transfer functions

$$
G_{i j}(s)=\left(E_{i} \frac{s^{i}}{\beta_{i}(s)}\right) P(s)^{-1}\left(D_{j} \beta_{j}(s)\right) .
$$

Extending Theorem 3.8 in [17] to higher order implicit difference equations, we obtain the following result.

THEOREM 4.7. Suppose that equation (1.5) is asymptotically stable and subjected to structured perturbations of the form 4.10). Then

$$
\begin{gathered}
\frac{1}{\max \left\{\sup _{|s| \in\{1, \infty\}}\left\|G_{i j}(s)\right\| \mid 0 \leq i, j \leq m\right\}} \leq r_{\mathbb{C}}^{b}(\underline{A}) \\
\leq \frac{1}{\max \left\{\sup _{|s| \in\{1, \infty\}}\left\|G_{i i}(s)\right\| \mid 0 \leq i \leq m\right\}}
\end{gathered}
$$

In particular, if $D_{i}=c_{i} D, 0 \neq c_{i} \in \mathbb{C}\left(\right.$ or $\left.E_{i}=c_{i} E\right)$ for all $i=0, \ldots, m$, then

$$
r_{\mathbb{C}}^{b}(\underline{A})=\frac{1}{\max \left\{\sup _{|s| \in\{1, \infty\}}\left\|G_{i i}(s)\right\| \mid 0 \leq i \leq m\right\}} .
$$

Proof. For $i=0, \ldots, m$, we restrict the blockdiagonal perturbation $\Delta_{b}$ such that $\Delta_{j}=0$ for all $j \neq i$. Then it is easy to see that the perturbation 4.10 can be rewritten in the form $\underline{\widetilde{A}}=\underline{A}+D_{i} \Delta_{i} \widehat{E}_{i}$, with $\widehat{E}_{i}=\left[0_{q_{i}, n(m-i)}, E_{i}, 0_{q_{i}, n i}\right]$. Using the formula for the stability radius in Theorem 3.2 it follows that

$$
r_{\mathbb{C}}^{b}(\underline{A}) \leq r_{\mathbb{C}}^{D_{i}, \widehat{E}_{i}}(\underline{A})=\frac{1}{\sup _{|s| \in\{1, \infty\}}\left\|G_{i i}(s)\right\|}
$$

Since this inequality holds for all $i=0, \ldots, m$, we obtain the second inequality in 4.12 .

To prove the first inequality in 4.12, let $\Delta_{b}=\operatorname{diag}\left(\Delta_{m}, \Delta_{m-1}, \ldots, \Delta_{0}\right)$ be a disturbance which makes the system non-regular or not asymptotical stable. Then there exists $s_{0} \in \mathbb{C} \backslash \mathbb{S}_{1}$ and a non-zero vector $y_{0} \in \mathbb{C}^{n}$ such that

$$
\widetilde{P}\left(s_{0}\right) y_{0}=\left(\sum_{i=0}^{m}\left(A_{i}+D_{i} \Delta_{i} E_{i}\right) s_{0}^{i}\right) y_{0}=P\left(s_{0}\right) y_{0}+\left(\sum_{i=0}^{m} D_{i} \Delta_{i} E_{i} s_{0}^{i}\right) y_{0}=0 .
$$

Since $P\left(s_{0}\right)$ is invertible, it follows that

$$
-P\left(s_{0}\right)^{-1}\left(\sum_{i=0}^{m} D_{i} \beta_{i}\left(s_{0}\right) \Delta_{i} E_{i} \frac{s_{0}^{i}}{\beta_{i}\left(s_{0}\right)}\right) y_{0}=y_{0} .
$$


Let $i_{0}$ be an index such that

$$
\left\|\left(E_{i_{0}} \frac{s_{0}^{i_{0}}}{\beta_{i_{0}}\left(s_{0}\right)}\right) y_{0}\right\|=\max \left\{\left\|\left(E_{i} \frac{s_{0}^{i}}{\beta_{i}\left(s_{0}\right)}\right) y_{0}\right\| \mid 0 \leq i \leq m\right\}
$$

then 4.14 implies that $\left(E_{i_{0}} \frac{s_{0}^{i_{0}}}{\beta_{i_{0}}\left(s_{0}\right)}\right) y_{0} \neq 0$. Multiplying 4.14 with $E_{i_{0}} \frac{s_{0}^{i_{0}}}{\beta_{i_{0}}\left(s_{0}\right)}$ from the left and taking norms, we obtain

$$
\sum_{j=0}^{m}\left\|G_{i_{0} j}\left(s_{0}\right)\right\|\left\|\Delta_{j}\right\|\left\|\left(E_{j} \frac{s_{0}^{j}}{\beta_{j}\left(s_{0}\right)}\right) y_{0}\right\| \geq\left\|\left(E_{i_{0}} \frac{s_{0}^{i_{0}}}{\beta_{i_{0}}\left(s_{0}\right)}\right) y_{0}\right\| .
$$

Hence,

$$
\begin{aligned}
\left\|\Delta_{b}\right\| & =\sum_{j=0}^{m}\left\|\Delta_{j}\right\| \geq \frac{1}{\max \left\{\left\|G_{i_{0} j}\left(s_{0}\right)\right\| \mid 0 \leq j \leq m\right\}} \\
& \geq \frac{1}{\max \left\{\left\|G_{i j}\left(s_{0}\right)\right\| \mid 0 \leq i, j \leq m\right\}} \\
& \geq \frac{1}{\sup _{s \in \mathbb{C} \backslash \mathbb{S}_{1}} \max \left\{\left\|G_{i j}(s)\right\| \mid 0 \leq i, j \leq m\right\}} \\
& =\frac{1}{\sup _{|s| \in\{1, \infty\}} \max \left\{\left\|G_{i j}(s)\right\| \mid 0 \leq i, j \leq m\right\}} \\
& =\frac{1}{\max \left\{\sup _{|s| \in\{1, \infty\}}\left\|G_{i j}(s)\right\| \mid 0 \leq i, j \leq m\right\}}
\end{aligned}
$$

Therefore, also the first inequality in 4.12 holds. If $D_{i}=c_{i} D$ then with $\beta_{i}(s)=1 / c_{i}$ for all $i=0, \ldots, m$ we obtain formula 4.13). If $E_{i}=c_{i} E$ then we choose $\beta_{i}(s)=s^{i} c_{i}$, $i=0, \ldots, m$ in 4.11 and obtain formula (4.13).

EXAMPLE 4.8. Consider the first order implicit difference equation $A_{1} x^{k+1}+$ $A_{0} x^{k}=0$ with $A_{1}=\left[\begin{array}{ll}1 & 1 \\ 0 & 0\end{array}\right], A_{0}=\left[\begin{array}{cc}1 & 0 \\ -1 & 1\end{array}\right]$. It is easy to see that the system is strangeness-free. Assume that the system is perturbed to the form

$$
\widetilde{A}_{1}=\left[\begin{array}{cc}
1+\delta_{1} & 1+\delta_{2} \\
0 & 0
\end{array}\right]=A_{1}+D_{1} \Delta_{1} E, \widetilde{A}_{0}=\left[\begin{array}{cc}
1 & 0 \\
-1+\delta_{3} & 1+\delta_{4}
\end{array}\right]=A_{0}+D_{0} \Delta_{0} E
$$

where

$$
D_{1}=\left[\begin{array}{l}
1 \\
0
\end{array}\right], D_{0}=\left[\begin{array}{l}
0 \\
1
\end{array}\right], E=I, \Delta_{1}=\left[\begin{array}{ll}
\delta_{1} & \delta_{2}
\end{array}\right], \Delta_{0}=\left[\begin{array}{ll}
\delta_{3} & \delta_{4}
\end{array}\right], \Delta=\left[\begin{array}{ll}
\delta_{1} & \delta_{2} \\
\delta_{3} & \delta_{4}
\end{array}\right] .
$$

The spectrum of the matrix polynomial

$$
P(s)=\left[\begin{array}{cc}
s+1 & s \\
-1 & 1
\end{array}\right]
$$

satisfies $\sigma(P)=\left\{-\frac{1}{2}\right\} \subset \mathbb{S}_{1}$ and thus this system is asymptotically stable. By simple algebraic manipulations we get

$$
H(s)=E P(s)^{-1} D(s)=\left[\begin{array}{cc}
\frac{1}{2 s+1} & \frac{-s}{2 s+1} \\
\frac{1}{2 s+1} & \frac{s+1}{2 s+1}
\end{array}\right]\left[\begin{array}{ll}
s & 0 \\
0 & 1
\end{array}\right]=\left[\begin{array}{cc}
\frac{s}{2 s+1} & \frac{-s}{2 s+1} \\
\frac{s}{2 s+1} & \frac{s+1}{2 s+1}
\end{array}\right] .
$$


Then, with respect to the maximum norm, we have $\sup _{|s| \in\{1, \infty\}}\|H(s)\|=2$. Hence, Theorem 4.5 we obtain

$$
r_{\mathbb{C}}(\underline{A}, D, E)=\frac{1}{2}
$$

and moreover, if the perturbation satisfies $\|\Delta\|<\frac{1}{2}$ then the perturbed system is also strangeness-free. In 4.15 we have restricted perturbations to the first row of the matrix $A_{1}$. If the second row of $A_{1}$ is perturbed, e.g.

$$
\widetilde{A}_{1}=\left[\begin{array}{cc}
1+\delta_{1} & 1+\delta_{2} \\
\delta_{1} & \delta_{2}
\end{array}\right]
$$

then it is easy to see that for every small enough $\epsilon \neq 0$, under the perturbations

$$
\Delta_{\epsilon}=\left[\begin{array}{ll}
\delta_{1} & \delta_{2} \\
\delta_{3} & \delta_{4}
\end{array}\right]=\left[\begin{array}{ll}
0 & \epsilon \\
0 & 0
\end{array}\right]
$$

the perturbed system has only finite eigenvalues and is not asymptotically stable.

EXAMPLE 4.9. Consider the second order implicit difference equation $A_{2} x^{k+2}+$ $A_{1} x^{k+1}+A_{0} x^{k}=0$ with $A_{2}, A_{1}, A_{0}$ given in Example 3.8. Suppose that the perturbed system is of the form

$$
\begin{aligned}
& \widetilde{A}_{2}=\left[\begin{array}{cc}
2+\delta_{1} & 0 \\
\delta_{2} & 0
\end{array}\right]=A_{2}+D_{2} \Delta_{2} E_{2}, \widetilde{A}_{1}=\left[\begin{array}{cc}
\delta_{3} & \delta_{4} \\
\delta_{3} & 2+\delta_{4}
\end{array}\right]=A_{1}+D_{1} \Delta_{1} E_{1} \\
& \widetilde{A}_{0}=\left[\begin{array}{cc}
-1+\delta_{5} & \delta_{5} \\
\delta_{6} & -1+\delta_{6}
\end{array}\right]=A_{0}+D_{0} \Delta_{0} E_{0}
\end{aligned}
$$

where $D_{2}=D_{0}=I, D_{1}=\left[\begin{array}{l}1 \\ 1\end{array}\right], E_{2}=\left[\begin{array}{ll}1 & 0\end{array}\right], E_{1}=I, E_{0}=\left[\begin{array}{ll}1 & 1\end{array}\right]$ and

$$
\Delta_{2}=\left[\begin{array}{l}
\delta_{1} \\
\delta_{2}
\end{array}\right], \Delta_{1}=\left[\begin{array}{ll}
\delta_{3} & \delta_{4}
\end{array}\right], \Delta_{0}=\left[\begin{array}{l}
\delta_{5} \\
\delta_{6}
\end{array}\right] .
$$

We choose $\beta_{i}(s)=1$ in 4.11 for all $s \in \mathbb{C} \backslash \mathbb{S}_{1}$ and obtain

$$
P(s)=\left[\begin{array}{cc}
2 s^{2}-1 & 0 \\
0 & 2 s-1
\end{array}\right], G_{i j}(s)=s^{i} E_{i} P(s)^{-1} D_{j},
$$

for $i, j=0,1,2$. With respect to the Euclidean norm, it follows that

$$
\sup _{|s| \in\{1, \infty\}}\left\|G_{i j}(s)\right\|= \begin{cases}2 & \text { if }(i, j) \in\{(0,1)\} \\ \sqrt{2} & \text { if }(i, j) \in\{(0,0),(0,2),(1,1)\} \\ 1 & \text { if }(i, j) \in\{(1,0),(1,2),(2,0),(2,1),(2,2)\} .\end{cases}
$$

Thus, by Theorem 4.7, we obtain

$$
\frac{1}{2} \leq r_{\mathbb{C}}^{b}(\underline{A}) \leq \frac{1}{\sqrt{2}} .
$$


5. Conclusion. Characterizations for asymptotic stability and robust asymptotic stability of higher order implicit difference equations have been presented under the assumption that the coefficient matrices are subjected to structured perturbations. Formulas for the real and complex stability radii have been derived and the equality of the real and complex stability radii has been studied for the class of strangeness-free and positive equations under admissible perturbations.

Acknowledgments: The second author was partially supported by a grant from the Niels Henrik Abel Board. This work was done while the second author was visiting at Vietnam Institute for Advance Study in Mathematics (VIASM) and Technische Universität Berlin. The second author would like to thank these instituitions for providing a fruitful research environment and hospitality. The first author was supported by DFG Collaborative Research Centre 910, Control of self-organizing nonlinear systems: Theoretical methods and concepts of application.

\section{REFERENCES}

[1] A. Berman and R.J. Plemmons, Nonnegative matrices in the mathematical sciences, Academic Press, New York, 1979.

[2] K. E. Brenan, S. L. Campbell, and L. R. Petzold, Numerical solution of initial-value problems in differential algebraic equations, 2nd ed., SIAM Publications, Philadelphia, PA, 1996.

[3] T. Brüll, Existence and uniqueness of solutions of linear variable coefficient discrete-time descriptor systems, Lin. Alg. Appl. 431 (2009), 247-265.

[4] _ Explixit solutions of regular linear discrete-time descriptor systems with constant coefficients, Electr. J. Lin. Alg. 18 (2009), 317-338.

[5] R. Byers and N.K. Nichols, On the stability radius of a generalized state-space system, Lin. Alg. Appl. 188-189 (1993), 113-134.

[6] S. L. Campbell, Singular systems of differential equations I, Pitman, San Francisco, CA, 1980.

[7] L. Dai, Singular control systems, Springer-Verlag, Berlin-Heidelberg, 1989.

[8] N.H. Du, V. H. Linh, and V. Mehrmann, Robust stability of differential-algebraic equations, In: Surveys in Differential-Algebraic Equations I, DAE Forum (2013), 63-95.

[9] N.H. Du, V.H. Linh, V. Mehrmann, and D.D.Thuan, Stability and robust stability of linear time-invariant delay differential-algebraic equations, SIAM J. Matr. Anal. Appl. 34 (2013), $1631-1654$.

[10] L. Farina and S. Rinaldi, Positive linear systems: Theory and applications, Nelson-Wiley, New York, 2000.

[11] F.R. Gantmacher, The theory of matrices II, Chelsea Publishing Company, New York, NY, 1959.

[12] I. Gohberg, P. Lancaster, and L. Rodman, Matrix polynomials, Academic Press, New York, 1982.

[13] P.M. Gy, Sampling of heterogeneous and dynamic material systems: Theories of heterogeneity, sampling and homogenizing, Elsevier Publications, Amsterdam, Netherlands, 1992.

[14] P. Ha and V. Mehrmann, Analysis and reformulation of linear delay differential-algebraic equations, Electr. J. Lin. Alg. 23 (2012), 703-730.

[15] D. Hinrichsen and A. J. Pritchard, Real and complex stability radii: A survey, In: D. Hinrichsen, B. Martensson eds., Control of Uncertain systems, Progress in System and Control Theory (1990), 119-162.

[16] D. Hinrichsen and N.K. Son, Stability radii of positive discrete-time systems under affine parameter perturbations, Int. J. Robust Nonlinear Control 8 (1998), 1169-1188.

[17] D. Hinrichsen, N.K. Son, and P.H.A. Ngoc, Stability radii of higher order positive difference systems, Systems Control Lett. 49 (2003), 377-388.

[18] P. Kunkel and V. Mehrmann, Differential-algebraic equations. Analysis and numerical solution, EMS Publishing House, Zürich, Switzerland, 2006.

[19] L. Lang, W. Chen, B.R. Bakshi, P.K. Goel, and S. Ungarala, Bayesian estimation via sequential monte carlo sampling - constrained dynamic systems, Automatica 43 (2007), 1615-1622.

[20] S. Lang, Complex analysis, Springer Verlag, New York, N.Y., 1999.

[21] J. P. LaSalle, The stability of dynamical systems, SIAM Publications, Philadelphia, PA, 1976.

[22] V. Mehrmann, R. Nabben, and E. Virnik, Generalisation of the Perron-Frobenius theory to matrix pencils, Lin. Alg. Appl. 428 (2008), 20-38. 
[23] V. Mehrmann and C. Shi, Transformation of high order linear differential-algebraic systems to first order, Numer. Alg. 42 (2006), 281-307.

[24] J.M. Ortega, Stability of difference equations and convergence of iterative processes, SIAM J. Numer. Anal. 10 (1973), 268-282.

[25] G. Pappas and D. Hinrichsen, Robust stability of linear systems described by higher order dynamic equations, IEEE Trans. Automat. Control 38 (1993), 1430-1435.

[26] L. Qiu, B. Bernhardsson, A. Rantzer, E.J. Davison, P.M. Young, and J.C. Doyle, A formula for computation of the real stability radius, Automatica 31 (1995), 879-890.

[27] N.K. Son and D.D. Thuan, The structured distance to non-surjectivity and its application to calculating the controllability radius of descriptor systems, J. Math. Anal. Appl. $\mathbf{3 8 8}$ (2012), 272-281.

[28] F. Tisseur and N.J. Higham, Structured pseudospectra for polynomial eigenvalue problems, with applications, SIAM J. Matr. Anal. Appl. 23 (2001), 187-208.

[29] L.N. Trefethen, Pseudospectra of linear operators, SIAM Rev. 39 (1997), 383-406. 